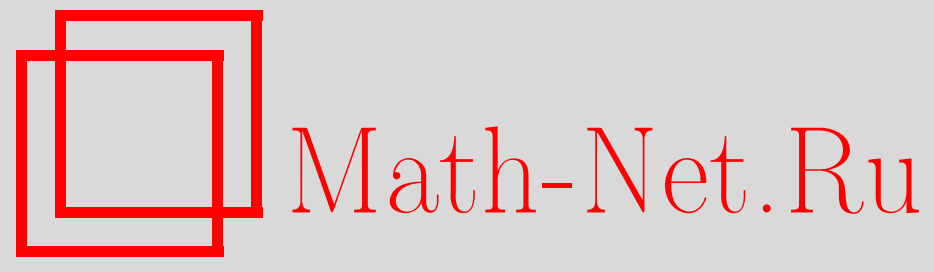

В. В. Волчков, Локальная теорема о двух радиусах для квазианалитических классов функций, Матем. заметкu, 2006, том 80, выпуск 4, 490-500

DOI: https://doi.org/10.4213/mzm2841

Использование Общероссийского математического портала Math-Net.Ru подразумевает, что вы прочитали и согласны с пользовательским соглашением http://www .mathnet.ru/rus/agreement

Параметры загрузки:

IP : 52.90 .164 .192

26 апреля 2023 г., 13:02:53

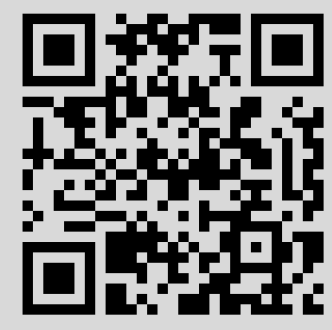




\section{ЛОКАЛЬНАЯ ТЕОРЕМА О ДВУХ РАДИУСАХ ДЛЯ КВАЗИАНАЛИТИЧЕСКИХ КЛАССОВ ФУНКЦИЙ}

\section{В. В. Волчков}

Получена точная характеристика максимальной гладкости ненулевых функций с нулевыми интегралами по шарам, радиусы которых принадлежат данному двухэлементному множеству.

Библиография: 24 названия.

Введение. Пусть $f$ - локально суммируемая функция на вещественном евклидовом пространстве $\mathbb{R}^{n}$ (обозначение $f \in L_{\mathrm{loc}}\left(\mathbb{R}^{n}\right)$ ), $E$ - заданное множество положительных чисел. Предположим, что при всех $r \in E$ и $x \in \mathbb{R}^{n}$

$$
\int_{|y| \leqslant r} f(x+y) d y=0
$$

где $|\cdot|$ - евклидова норма в $\mathbb{R}^{n}$. Для каких $E$ отсюда следует, что $f$ - нулевая функция? Известная теорема Л. Зальцмана о двух радиусах утверждает, что $f=0$, если $E$ состоит из двух положительных чисел $r_{1}$ и $r_{2}$, для которых число $r_{1} / r_{2}$ не является отношением корней функции Бесселя $J_{n / 2}$ (см. [1]). Примеры показывают (см. [1]), что указанное условие для $r_{1} / r_{2}$ является необходимым.

Получение локального аналога теоремы Зальцмана, например, когда $f$ задана в шаре $B_{R}=\left\{x \in \mathbb{R}^{n}:|x|<R\right\}, R>\max \left(r_{1}, r_{2}\right)$, и равенство (1) выполнено при всех $x \in B_{R-r}$, является существенно более трудной проблемой. Возникающее при этом нарушение групповой структуры сдвигов, действующей на пространстве решений (1), создает серьезные препятствия для применения методов анализа Фурье, использованных в [1]. Первые результаты в этом направлении содержатся в работах Смита, Беренстейна, Гэя, Ижера (см. [2]-[4]). Принципиально новый метод, основанный на явном решении уравнения (1) в специальных функциях, был предложен в [5], где получен окончательный вариант локальной теоремы о двух радиусах. Для его формулировки введем следующие обозначения: $\Lambda_{n}=\left\{\nu_{1}, \nu_{2}, \ldots\right\}$ - последовательность всех положительных корней функции $J_{n / 2}$, занумерованных в порядке возрастания; $E_{n}$ - множество чисел вида $\alpha / \beta$, где $\alpha, \beta \in \Lambda_{n}$;

$$
A_{n}=\left\{t>0: \forall N>0 \exists \alpha, \beta \in \Lambda_{n}:\left|t-\frac{\alpha}{\beta}\right|<(1+\beta)^{-N}\right\} .
$$

Работа выполнена при частичной поддержке фонда ISF, грант № U91200, фонда ISSEP, грант № APU071013, и фонда МНОП, грант № YSU081012.

(C) В.В. Волчков, 2006 
Структура множества $A_{n}$ подробно изучена в [5; $\S 8$. Отметим, что $A_{n}$ имеет нулевую лебегову меру на $(0,+\infty)$ и пересечение $A_{n}$ с любым интервалом $(a, b) \subset(0,+\infty)$ имеет мощность континуума. В работе [5] доказана

Teopema A. Пyсmь $E=\left\{r_{1}, r_{2}\right\}, R>\max \left(r_{1}, r_{2}\right), f \in L_{\mathrm{loc}}\left(B_{R}\right) u$

$$
\int_{|y| \leqslant r} f(x+y) d y=0 \quad \text { при всех } \quad r \in E, \quad x \in B_{R-r} .
$$

Тогда выполнены следующие утверждения:

1) если $r_{1}+r_{2}<R$ u $r_{1} / r_{2} \notin E_{n}$, mo $f=0$;

2) если $r_{1}+r_{2}=R, r_{1} / r_{2} \notin E_{n}, u f \in C^{\infty}\left(B_{R}\right)$, mo $f=0$;

3) если $n=1, r_{1}+r_{2}=R$ u $r_{1} / r_{2} \notin E_{1}$, mo $f=0$;

4) если $r_{1}+r_{2}=R$ u $r_{1} / r_{2} \in A_{n} \backslash E_{n}$, mo $f=0$;

5) если $n \geqslant 2, r_{1}+r_{2}=R$ u $r_{1} / r_{2} \notin A_{n}$, то для любого $m \in \mathbb{N}$ существует ненулевая $f \in C^{m}\left(B_{R}\right)$ с условием (2);

6) если $r_{1}+r_{2}>R$, то существует ненулевая $f \in C^{\infty}\left(B_{R}\right)$ с условием (2);

7) если $r_{1} / r_{2} \in E_{n}$, то существует ненулевая вещественноаналитическая на $\mathbb{R}^{n}$ функиия $f$, для которой равенство (2) выполнено при всех $x \in \mathbb{R}^{n}$.

Отметим, что утверждения 1), 2) и 7) теоремы А содержатся и в предшествующих работах [1]-[4]. При $R>r_{1}+r_{2}+\min \left(r_{1}, r_{2}\right)$ первое утверждение доказано Смитом [2]. Различные доказательства утверждений 1), 2) были даны Беренстейном, Гэем и Ижером (см. [3], [4]). Утверждение 6) при дополнительном ограничении $r_{1} / r_{2} \notin A_{n}$ получено в [3].

Утверждения 5), 2) и 7) теоремы А позволяют сделать вывод о характере максимальной гладкости ненулевых функций, удовлетворяющих (2) при соответствующих $r_{1}, r_{2}$. В частности, наибольшая степень гладкости (вещественная аналитичность) у таких функций достигается в случае $r_{1} / r_{2} \in E_{n}$. При условиях утверждения 5) допустима любая конечная гладкость и этот результат является неулучшаемым (см. утверждение 2)). В остальных случаях, т.е. при $r_{1} / r_{2} \notin E_{n}$ и $r_{1}+r_{2}>R$, вопрос о точной характеристике максимальной гладкости $f \neq 0$, удовлетворяющей (2), оставался открытым. В данной работе получено решение этой задачи в терминах теории квазианалитических классов функций.

1. Формулировка основного результата. Пусть $\mathbb{Z}_{+}^{n}-$ множество $n$-мерных мультииндексов, т.е. всех векторов из $\mathbb{R}^{n}$ с неотрицательными целыми координатами. Для мультииндекса $\alpha=\left(\alpha_{1}, \ldots, \alpha_{n}\right)$ с длиной $|\alpha|=\sum_{j=1}^{n} \alpha_{j}$ обозначим $\partial^{\alpha}=\partial^{|\alpha|} /\left(\partial x_{1}^{\alpha_{1}} \cdots \partial x_{n}^{\alpha_{n}}\right)$ - операторы частного дифференцирования порядка $|\alpha|$. Пусть также $\bar{G}$ - замыкание области $G \subset \mathbb{R}^{n}$.

Основным результатом данной работы является

Tеорема 1. Пусть $E=\left\{r_{1}, r_{2}\right\}, R>r_{2}>r_{1}, r_{1}+r_{2}>R, f \in C^{\infty}\left(B_{R}\right) u$ выполнено условие (2). Тогда имеют место следующие утверждения:

1) если $r_{1} / r_{2} \notin E_{n}$ и существует последовательность положительных чисел $\left\{M_{q}\right\}_{q=0}^{\infty}$ такая, что

$$
\sum_{j=1}^{\infty} \frac{1}{\inf _{q \geqslant j} M_{q}^{1 / q}}=\infty
$$


и для любого $\alpha \in \mathbb{Z}_{+}^{n}$

$$
\sup _{x \in B_{r_{1}}}\left|\left(\partial^{\alpha} f\right)(x)\right| \leqslant M_{|\alpha|},
$$

$m o f=0$;

2) для любой последовательности положительных чисел $\left\{M_{q}\right\}_{q=0}^{\infty}$ такой, что

$$
\sum_{j=1}^{\infty} \frac{1}{\inf _{q \geqslant j} M_{q}^{1 / q}}<\infty,
$$

существует ненулевая функиия $f \in C^{\infty}\left(B_{R}\right)$, удовлетворяющая (2), у которой

$$
\sup _{x \in B_{R}}\left|\left(\partial^{\alpha} f\right)(x)\right| \leqslant M_{|\alpha|}
$$

при всех $\alpha \in \mathbb{Z}_{+}^{n}$.

Согласно теореме Карлемана (см. п. 2) условия (3), (4) означают, что $f$ принадлежит квазианалитическому классу функций в шаре $\bar{B}_{r_{1}}$. В частности, при $r_{1} / r_{2} \in E_{n}$ первое утверждение теоремы 1 неверно (см. утверждение 7) теоремы А). Отметим также, что в неравенстве (4) нельзя заменить $B_{r_{1}}$ открытым шаром меньшего радиуса с центром в нуле (см. п. 4). Таким образом, точной характеристикой максимально допустимой гладкости $f \neq 0$, удовлетворяющей (2) при $r_{1} / r_{2} \notin E_{n}, r_{1}+r_{2}>R$, является отсутствие квазианалитичности в $\bar{B}_{r_{1}}$.

По поводу других результатов, связанных с теоремой о двух радиусах, см. [6]--[18] и библиографию к этим работам.

Автор благодарит Л. Зальцмана и К. А. Беренстейна за обсуждения и представленную литературу.

2. Обозначения и вспомогательные конструкции. Пусть $G$ - область в $\mathbb{R}^{n}$ и $\mathscr{M}=\left\{M_{q}\right\}_{q=0}^{\infty}-$ заданная последовательность положительных чисел. Обозначим через $C^{\mathscr{M}}(\bar{G})$ множество всех функций $f \in C^{\infty}(\bar{G})$ таких, что

$$
\left|\left(\partial^{\alpha} f\right)(x)\right| \leqslant K^{|\alpha|+1} M_{|\alpha|} \quad \text { при всех } \quad x \in \bar{G}, \quad \alpha \in \mathbb{Z}_{+}^{n},
$$

где постоянная $K>0$ зависит от $f$, но не от $\alpha$ и $x$. Класс $C^{\mathscr{M}}(\bar{G})$ называется квазианалитическим, если для всякой функции $f \in C^{\mathscr{M}}(\bar{G})$ из условия $\left(\partial^{\alpha} f\right)\left(x_{0}\right)=0$ при некотором $x_{0} \in \bar{G}$ и всех $\alpha \in \mathbb{Z}_{+}^{n}$ следует, что $f=0$. Известная теорема Карлемана (обычно формулируемая для случая отрезка на $\mathbb{R}^{1}$, из которого легко следует общий случай) утверждает, что для квазианалитичности класса $C^{\mathscr{M}}(\bar{G})$ необходимо и достаточно выполнения условия (3) (см., например, [19], [20; гл. 1] и библиографию к этим работам).

Для фиксированного $r>0$ и $R>r$ обозначим $V_{r}\left(B_{R}\right)$ множество функций $f \in C^{\infty}\left(B_{R}\right)$, имеющих нулевые интегралы по всем замкнутым шарам радиуса $r$, содержащимся в $B_{R}$. Пусть также $V_{r}^{\mathscr{M}}\left(B_{R}\right)$ - множество всех функций $f \in V_{r}\left(B_{R}\right)$ таких, что сужение $f$ на $\bar{B}_{r}$ принадлежит $C^{\mathscr{M}}\left(\bar{B}_{r}\right)$.

Всюду в дальнейшем, если не оговорено противного, предполагается, что $n \geqslant 2$. Пусть $S^{n-1}=\left\{x \in \mathbb{R}^{n}:|x|=1\right\}, \rho, \sigma-$ полярные координаты в $\mathbb{R}^{n}$ (для любого $x \in \mathbb{R}^{n} \quad \rho=|x|$, а если $x \neq 0$, то $\left.\sigma=x / \rho \in S^{n-1}\right)$. Обозначим: $\mathscr{H}_{k}-$ пространство сферических гармоник степени $k$ на $S^{n-1}$, рассматриваемое как подпространство $L^{2}\left(S^{n-1}\right)$ (см. $[21 ;$ гл. $\left.4, \S 2]\right), d_{k}$ - размерность $\mathscr{H}_{k},\left\{Y_{l}^{(k)}\right\}, 1 \leqslant l \leqslant d_{k},-$ 
фиксированный ортонормированный базис в $\mathscr{H}_{k}$. Всякой функции $f \in L_{\mathrm{loc}}\left(B_{R}\right)$ соответствует ряд Фурье

$$
f(x) \sim \sum_{k=0}^{\infty} \sum_{l=1}^{d_{k}} F_{k, l}(\rho) Y_{l}^{(k)}(\sigma), \quad \rho \in(0, R),
$$

где

$$
f_{k, l}(\rho)=\int_{S^{n-1}} f(\rho \sigma) \overline{Y_{l}^{(k)}(\sigma)} d \sigma .
$$

Пусть $\mathrm{SO}(n)$ - группа вращений $\mathbb{R}^{n}$ с нормированной мерой Хаара $d \tau, T^{k}(\tau)$ сужение квазирегулярного представления группы $\mathrm{SO}(n)$ на пространство $\mathscr{H}_{k}$ (см. $[22 ;$ гл. $9, \S 2$, п. 7$]),\left\{t_{l, p}^{k}\right\}, 1 \leqslant l, p \leqslant d_{k},-$ матрица представления $T^{k}(\tau)$, т.е.

$$
Y_{l}^{(k)}\left(\tau^{-1} \sigma\right)=\sum_{p=1}^{d_{k}} t_{l, p}^{k}(\tau) Y_{p}^{(k)}(\sigma), \quad \tau \in \mathrm{SO}(n), \quad \sigma \in S^{n-1} .
$$

В частности, при $k=0$ имеем $d_{0}=1, Y_{1}^{(0)}(\sigma)=1, t_{1,1}^{0}(\tau)=1$ при всех $\sigma \in S^{n-1}$, $\tau \in \mathrm{SO}(n)$. При $n=2$ и $k \geqslant 1$ всюду в дальнейшем будет использоваться следующий базис в $\mathscr{H}_{k}$ :

$$
Y_{1}^{(k)}(\sigma)=\left(\sigma_{1}+i \sigma_{2}\right)^{k}, \quad Y_{2}^{(k)}(\sigma)=\left(\sigma_{1}-i \sigma_{2}\right)^{k}
$$

(в этом случае $d_{k}=2$ при всех $k \geqslant 1$ ). Если $\tau$ - вращение на угол $\theta$ в $\mathbb{R}^{2}$, то для этого базиса

$$
t_{1,1}^{k}(\tau)=\overline{t_{2,2}^{k}(\tau)}=e^{-i k \theta}, \quad t_{1,2}^{k}(\tau)=t_{2,1}^{k}(\tau)=0
$$

При этом для членов ряда (8) из формулы (9) имеем равенство

$$
f_{k, l}(\rho) Y_{l}^{(k)}(\delta)=\int_{\mathrm{SO}(2)} f\left(\tau^{-1} x\right) \overline{t_{l, l}^{k}(\tau)} d \tau
$$

При $n \geqslant 3$ из неприводимости $T^{k}(\tau)$ (см. [22; гл. $9, \S 2$, п. 10]) следует, что при всех $1 \leqslant l, p \leqslant d_{k}$

$$
f_{k, l}(\rho) Y_{p}^{(k)}(\sigma)=d_{k} \int_{\mathrm{SO}(n)} f\left(\tau^{-1} x\right) \overline{t_{l, p}^{k}(\tau)} d \tau
$$

(см. [10; гл. 9, §2, п. 10]). Формулы $(10),(11)$ показывают, что для $f \in C^{\infty}\left(B_{R}\right)$ члены ряда (8) можно доопределить в точке $x=0$ так, что они станут функциями класса $C^{\infty}\left(B_{R}\right)$. Далее все функции из класса $C^{\infty}$ в проколотой окрестности нуля в $\mathbb{R}^{n}$, допускающие такое продолжение в точку $x=0$, считаются доопределенными в нуле указанным образом.

\section{3. Свойства функций класса $V_{r}^{\mathscr{M}}\left(B_{R}\right)$.}

Лемма 1. Пусть $f \in V_{r}^{\mathscr{M}}\left(B_{R}\right)$. Тогда для любъх $k \in \mathbb{Z}_{+}, 1 \leqslant l, p \leqslant d_{k}$

$$
f_{k, l}(\rho) Y_{p}^{(k)}(\sigma) \in V_{r}^{\mathscr{M}}\left(B_{r}\right) .
$$


Доказательство. Пусть сначала $n \geqslant 3, \tau \in \mathrm{SO}(n)$ и $\left\{\xi_{i, j}(\tau)\right\}, 1 \leqslant i, j \leqslant n,-$ opтогональная матрица, соответствующая вращению $\tau$. Полагая $u(x)=f_{k, l}(\rho) Y_{p}^{(k)}(\sigma)$, из (11) получаем

$$
\begin{aligned}
\left(\partial^{\alpha} u\right)(x)=d_{k} & \int_{\mathrm{SO}(n)} \sum_{s_{1}=1}^{n} \cdots \sum_{s_{|\alpha|}=1}^{n} \xi_{s_{1}, j_{1}}(\tau) \cdots \xi_{s_{|\alpha|}, j_{|\alpha|}}(\tau) \\
& \times\left(\frac{\partial^{|\alpha|} f}{\partial x_{s_{1}} \cdots \partial x_{s_{|\alpha|}}}\right)\left(\tau^{-1} x\right) \overline{t_{l, p}^{k}(\tau)} d \tau
\end{aligned}
$$

для некоторых $j_{1}, \ldots, j_{|\alpha|}$, зависящих только от $\alpha \in \mathbb{Z}_{+}^{n} \backslash\{0\}$. Поскольку $\left|\xi_{i, j}(\tau)\right| \leqslant 1$, из неравенства (7) для $G=B_{r}$ и $[22 ;$ гл. $1, \S 4$, п. 3 , формула (3)] имеем оценку

$$
\left|\left(\partial^{\alpha} u\right)(x)\right| \leqslant K^{|\alpha|+1} M_{|\alpha|}, \quad x \in \bar{B}_{r}, \quad \alpha \in \mathbb{Z}_{+}^{n},
$$

где постоянная $K>0$ не зависит от $\alpha$ и $x$. Кроме того, $u \in V_{r}\left(B_{r}\right)$ (см. [5; лемма 1]). Отсюда следует утверждение леммы при $n \geqslant 3$. Аналогичные рассуждения (с использованием (10) вместо (11)) позволяют доказать лемму 1 и при $n=2, p=l$. Пусть теперь $n=2, p \neq l$. Очевидно, если $f\left(x_{1}, x_{2}\right) \in V_{r}^{\mathscr{M}}\left(B_{R}\right)$, то $f\left(x_{1},-x_{2}\right) \in V_{r}^{\mathscr{M}}\left(B_{R}\right)$. Поскольку $Y_{2}^{(k)}\left(\sigma_{1}, \sigma_{2}\right)=Y_{1}^{(k)}\left(\sigma_{1},-\sigma_{2}\right)$, утверждение следует из уже рассмотренных случаев. Таким образом, лемма 1 доказана.

Лемма 2. Пустъ $f \in V_{r}^{\mathscr{M}}\left(B_{R}\right), s \in \mathbb{N} u \mathscr{M}(s)=\left\{M_{q+s}\right\}_{q=0}^{\infty}$. Тогда имеют место следующие утверждения:

1) $\partial^{\beta} f \in V^{\mathscr{M}(|\beta|)}\left(B_{R}\right)$ для любого $\beta \in \mathbb{Z}_{+}^{n}$;

2) при всех $k \in \mathbb{N}, 1 \leqslant l \leqslant d_{k}$

$$
\left(D_{k} f_{k, l}\right)(\rho) \in V^{\mathscr{M}(k)}\left(B_{R}\right)
$$

где

$$
D_{k}=\prod_{j=1}^{n}\left(\frac{d}{d \rho}+\frac{n+k-j-1}{\rho} I\right),
$$

I - тождественный оператор.

ДоКАЗАТЕЛЬСтво. Первое утверждение непосредственно следует из определения класса $V_{r}^{\mathscr{M}}\left(B_{R}\right)$. Докажем утверждение 2). По лемме 1 функция $u(x)=$ $f_{k, l}(\rho) Y(\sigma) \in V_{r}^{\mathscr{M}}\left(B_{R}\right)$ при любом $Y \in \mathscr{H}_{k}$. Полагая

$$
P(x)=\rho^{k} Y(\sigma), \quad Q(x)=(n+2 k-2) x_{1} P-\rho^{2} \frac{\partial P}{\partial x_{1}},
$$

получаем

$$
(n+2 k-2) \frac{\partial u}{\partial x_{1}}=\left(f_{k, l}^{\prime}(\rho)-\frac{k}{\rho} f_{k, l}(\rho)\right) Q(x) \rho^{-k-1}+\left(f_{k, l}^{\prime}(\rho)+\frac{n+k-2}{\rho} f_{k, l}(\rho)\right) \rho^{1-k} \frac{\partial P}{\partial x_{1}} .
$$

Поскольку $\rho^{-k-1} Q \in \mathscr{H}_{k+1}, \rho^{1-k} \frac{\partial P}{\partial x_{1}} \in \mathscr{H}_{k-1}$ (см. [22; гл. 9, $\S 2$, п. 3]), из утверждения 1) и леммы 1 имеем

$$
\left(f_{k, l}^{\prime}(\rho)+\frac{n+k-2}{\rho} f_{k, l}(\rho)\right) Y^{(k-1)}(\sigma) \in V_{r}^{\mathscr{M}(1)}\left(B_{R}\right)
$$

при любом $Y^{(k-1)} \in \mathscr{H}_{k-1}$. Проведя подобное рассуждение $k$ раз, получим (12). 
ЛЕмма 3. Пусть $n \geqslant 1 u$

$$
\varphi_{n}(x)=J_{(n-2) / 2}(|x|)|x|^{(2-n) / 2}, \quad x \in \mathbb{R}^{n} .
$$

Тогда имеют место следующие утверждения:

1) для всех $\alpha \in \mathbb{Z}_{+}^{n}, x \in \mathbb{R}^{n}$

$$
\left|\left(\partial^{\alpha} \varphi_{n}\right)(x)\right| \leqslant \frac{2^{1-n / 2}}{\Gamma(n / 2)},
$$

где $\Gamma(\cdot)$ - гамма-функиия;

2) при любых $r>0, y \in \mathbb{R}^{n}$

$$
\int_{|x| \leqslant r} \varphi_{n}(x+y) d x=(2 \pi r)^{n / 2} J_{n / 2}(r) \varphi_{n}(y) .
$$

ДОКАЗАТЕЛЬСТВО. При $n=1$ имеем

$$
\varphi_{1}(x)=J_{-1 / 2}(|x|)|x|^{1 / 2}=\sqrt{\frac{2}{\pi}} \cos x
$$

(см. [23; гл. $1, \S 7])$. В этом случае утверждения лемм 3 очевидны. Пусть $n \geqslant 2$. Тогда неравенство (14) вытекает из формулы

$$
\varphi_{n}(x)=\frac{1}{(2 \pi)^{n}} \int_{S^{n-1}} e^{i(x, \sigma)} d \sigma,
$$

где

$$
(x, \sigma)=\sum_{j=1}^{n} x_{j} \sigma_{j}
$$

(см., например, [24; введение, лемма 3.6]). Равенство (15) является следствием теоремы о среднем для решений уравнения Гельмгольца (см. [5; формула (12)]).

ЛЕмма 4. Пусть $\left\{a_{q}\right\}_{q=1}^{\infty}-$ последовательность положительных чисел такая, что $\sum_{q=1}^{\infty} a_{q}^{-1}=+\infty$. Тогда для любого $\gamma>0$

$$
\sum_{q=1}^{\infty} a_{q}^{-1-\gamma / q}=+\infty
$$

ДокАЗАТЕЛЬСтво. Обозначим $\mathscr{E}_{\gamma}=\left\{q \in \mathbb{N}: a_{q}^{\gamma}>2^{q}\right\}$ и предположим, что

$$
\sum_{q=1}^{\infty} a_{q}^{-1-\gamma / q}<+\infty
$$

Тогда

$$
\sum_{q=1}^{\infty} a_{q}^{-1}=\sum_{q \in \mathscr{E}_{\gamma}} a_{q}^{-1}+\sum_{q \notin \mathscr{E}_{\gamma}} a_{q}^{-1}<\sum_{q \in \mathscr{E}_{\gamma}} 2^{-q / \gamma}+2 \sum_{q \notin \mathscr{E}_{\gamma}} a^{-1-\gamma / q}<+\infty
$$

что противоречит условию. 
Лемма 5. Пусть $n \geqslant 1$ u $g(|x|) \in V_{r}^{\mathscr{M}}\left(B_{R}\right)$. Тогда при $x \in B_{R}$ выполнено равенство

$$
g(|x|)=\sum_{q=1}^{\infty} c_{q} \varphi_{n}\left(\frac{x}{r} \nu_{q}\right), \quad c_{q} \in \mathbb{C},
$$

где $\varphi_{n}$ определяется формулой (13). Кроме того, для любых $q \in \mathbb{N}, j \in \mathbb{Z}_{+}$имеет место оиенка

$$
\left|c_{q}\right| \leqslant K^{j+1} \nu^{n-1-j} M_{j}
$$

с постоянной $K>0$, не зависящей от $j u q$.

ДокАЗАТЕЛЬСТво. При $n=1$ из условия следует

$$
\int_{t-r}^{t+r} g(|x|) d x=0 \quad \text { при } \quad|t|<R-r .
$$

Дифференцируя по $t$, получаем, что $g(|x|)$ периодична с периодом $2 r$ на $(-R, R)$. Учитывая, что $\Lambda_{1}=\{\pi m\}_{m=1}^{\infty}$ (см. [23; гл. $\left.\left.1, \S 7\right]\right)$ и разлагая $g(|x|)$ в ряд Фурье на $[-r, r]$, из (6) имеем (17). Оценка (18) для коэффициентов Фурье следует из неравенства

$$
\left(\frac{d}{d x}\right)^{j}(g(|x|)) \leqslant K^{j+1} M_{j}, \quad x \in[-r, r],
$$

вытекающего из условия $g(|x|) \in V_{r}^{\mathscr{M}}\left(B_{R}\right)$.

Пусть теперь $n \geqslant 2$. Разложение (17) для функции $g(|x|) \in V_{r}\left(B_{R}\right)$, в котором $c_{q}=O\left(\nu_{q}^{-p}\right)$ при $q \rightarrow \infty$ и любом фиксированном $p>0$, получено в $[5 ; \S 7$, следствие из теоремы 3]. При этом условии ряд в (17) сходится равномерно на $\mathbb{R}^{n}$ и допускает почленное дифференцирование любое число раз (см. (14)). Используя [23; гл. 1 , формула (1.1)], а также [23; гл. 1 , формула (6.3)], из (17) и определения $\nu_{q}$ получаем

$$
\left.\frac{d}{d \rho}\left(\frac{d^{2}}{d \rho^{2}}+\frac{n-1}{\rho} \frac{d}{d \rho}\right)^{m}(g(\rho))\right|_{\rho=r}=0
$$

при любом $m \in \mathbb{Z}_{+}$. Докажем оценку (18). Поскольку

$$
\nu_{q} J_{(n-2) / 2}^{\prime}\left(\nu_{q}\right)-\frac{n-2}{2} J_{(n-2) / 2}\left(\nu_{q}\right)=-\nu_{q} J_{n / 2}\left(\nu_{q}\right)=0
$$

(см. [23; гл. 1, формула (6.3)]), равенство

$$
t^{(n-2) / 2} g(r t)=\sum_{q=1}^{\infty} c_{q} \nu_{q}^{(2-n) / 2} J_{(n-2) / 2}\left(\nu_{q} t\right)
$$

есть разложение функции $t^{(n-2) / 2} g(r t)$ в ряд Дини на $[0,1]$ (см. [23; гл. 2, §24]). Из $(21)$ и $[23 ;$ гл. 2 , формула $(24.10)]$ находим

$$
c_{q} J_{(n-2) / 2}^{2}\left(\nu_{q}\right)=2 \nu_{q}^{n / 2-1} \int_{0}^{1} t^{n / 2} g(r t) J_{(n-2) / 2}\left(\nu_{q} t\right) d t .
$$


Интегрируя по частям с учетом (19), (20) и [23; гл. 1, формула (6.2)], при любом $m \in \mathbb{Z}_{+}$имеем

$$
\begin{aligned}
c_{q} J_{(n-2) / 2}^{2}\left(\nu_{q}\right) \nu_{q}^{1-n / 2-2 m}\left(-r^{2}\right)^{m} & \\
\quad= & 2 \int_{0}^{1} t^{n / 2}\left(\left(\frac{d^{2}}{d t^{2}}+\frac{n-1}{t} \frac{d}{d t}\right)^{m} g\right)(r t) J_{(n-2) / 2}\left(\nu_{q} t\right) d t \\
& =\frac{-2}{\nu_{q}} \int_{0}^{1} t^{n / 2}\left(\frac{d}{d t}\left(\frac{d^{2}}{d t^{2}}+\frac{n-1}{t} \frac{d}{d t}\right)^{m} g\right)(r t) J_{n / 2}\left(\nu_{q} t\right) d t .
\end{aligned}
$$

Используя [24; введение, §3, формула (3)], а также [5; формулы (1), (11)], отсюда и из оценки (14) при $\alpha=0$ получаем (18).

\section{4. Доказательство теоремы 1.}

ДоКАЗАТЕЛЬСТво УТВЕРЖДЕНИЯ 1) тЕОРЕмы 1 . Пусть сначала $n \geqslant 2, f \in$ $C^{\infty}\left(B_{R}\right)$ и выполнены условия (2)-(4). Докажем, что $f_{k, l}=0$ при всех $k, l$. Положим

$$
g(\rho)=\left(D_{k} f_{k, l}\right)(\rho)
$$

тогда $g(\rho) \in V_{r}^{\mathscr{M}(k)}\left(B_{R}\right)$ (см. лемму 2). По лемме 5 при $r=r_{1}$ выполнено равенство (17), в котором

$$
\left|c_{q}\right| \leqslant K_{1}^{j+1} \nu_{q}^{n-1-j} M_{j+k}, \quad j \in \mathbb{Z}_{+},
$$

и постоянная $K_{1}>0$ не зависит от $j$ и $q$. Продолжим $g(|x|)$ на $\mathbb{R}^{n}$ по формуле (17) (это возможно ввиду (14), (23) и [5; формула (11)]). Тогда $g(|x|) \in V_{r_{1}}\left(\mathbb{R}^{n}\right)$ (см., например, (15)). Полагая

$$
h(x)=\int_{|y| \leqslant r_{2}} g(|x+y|) d y
$$

из (17) и (15) находим

$$
h(x)=\sum_{q=1}^{\infty} c_{q}\left(2 \pi r_{2}\right)^{n / 2}\left(\frac{r_{1}}{\nu_{q}}\right)^{n-1} J_{n / 2}\left(\frac{r_{2}}{r_{1}} \nu_{q}\right) \varphi_{n}\left(\frac{\nu_{q}}{r_{1}} x\right) .
$$

Используя (14), (23) и [5; формула (10)], отсюда имеем

$$
\left|\left(\partial^{\alpha} h\right)(x)\right| \leqslant K_{1}^{j+1} M_{j+k} \sum_{q=1}^{\infty} \nu_{q}^{|\alpha|-j-1 / 2}
$$

при любых $x \in \mathbb{R}^{n}, \alpha \in \mathbb{Z}_{+}^{n}, j>|\alpha|$ (постоянная $K_{1}>0$ не зависит от $x, j, \alpha$ ). Полагая в (24) $j=|\alpha|+1$ и учитывая [5; формула (11)], получаем оценку

$$
\left|\left(\partial^{\alpha} h\right)(x)\right| \leqslant K_{2}^{|\alpha|+1} M_{|\alpha|+1+k}, \quad x \in \mathbb{R}^{n},
$$

с константой $K_{2}>0$, не зависящей от $x$ и $\alpha$. Тогда из (3), леммы 4 и теоремы Карлемана (см. п. 2) следует, что $h$ принадлежит квазианалитическому классу функций в любом шаре $B \subset \mathbb{R}^{n}$. Поскольку $f \in V_{r_{2}}\left(B_{R}\right)$, из $(22)$ и леммы 2 получаем, что 
$h=0$ в $B_{R-r_{2}}$. Тогда $h=0$ в $\mathbb{R}^{n}$ и $g(\rho) \in V_{r}\left(R^{n}\right), r=r_{1}, r_{2}$. Так как $r_{1} / r_{2} \notin E_{n}$, из первого утверждения теоремы А вытекает, что $g=0$. Учитывая, что $f_{k, l}$ как функция переменной $\rho \in[0, R)$ принадлежит классу $C^{\infty}[0, R)($ см. (9)), отсюда и из $(22)$ имеем $f_{k, l}=0$. Таким образом, $f=0$ в $B_{R}$ и при $n \geqslant 2$ утверждение 1 ) доказано.

Пусть $n=1$. В этом случае положим $g(|x|)=f(x)+f(-x)$. Тогда $g(|x|) \in$ $V_{r}^{\mathscr{M}}(-R, R)$ и по лемме 5 при $r=r_{1}$ имеют место (17), (18). Повторяя приведенные выше рассуждения, получим $g=0$, откуда $f^{\prime}(x)=f^{\prime}(-x)$. Далее, для производной $f^{\prime} \in V_{r}^{\mathscr{M}(1)}(-R, R)$ аналогично имеем $f^{\prime}=0$. Поскольку $f \in V_{r}(-R, R)$, отсюда следует утверждение 1$)$ и при $n=1$.

ЗАмечаниЕ. Отметим, что утверждение 1) становится неверным, если в неравенстве (4) заменить $B_{r_{1}}$ открытым шаром меньшего радиуса с центром в нуле. Действительно, для любого $\varepsilon>0$ положим $R=r_{1}+\varepsilon / 2$. Тогда всякая функция $f \in C^{\infty}\left(\mathbb{R}^{n}\right)$ с носителем в $G=B_{r_{1}-\varepsilon / 2} \backslash \bar{B}_{r_{1}-\varepsilon}$ такая, что

$$
\int_{G} f(x) d x=0
$$

принадлежит $V_{r}\left(B_{R}\right)$ при всех $r \geqslant r_{1}$ и $\partial^{\alpha} f=0$ в $\bar{B}_{r_{1}-\varepsilon}$ для любого $\alpha \in \mathbb{Z}_{+}^{n}$.

ДОКАЗАТЕЛЬСТвО УТВЕРЖДЕНИЯ 2) тЕОРЕМЫ 1 . Пусть $r_{1}+r_{2}-R=\varepsilon_{1}>0$ и выполнены условия (5), (6). Не ограничивая общности, можно считать, что последовательность $\left\{M_{q}^{1 / q}\right\}_{q=1}^{\infty}$ не убывает (в противном случае $M_{q}$ можно заменить на $\left.m_{q}=\left(\inf _{j \geqslant q} M_{j}^{1 / j}\right)^{q} \leqslant M_{q}\right)$. Из (6) следует, что существует последовательность положительных чисел $\left\{\zeta_{q}\right\}_{q=0}^{\infty}$ такая, что

$$
\lim _{q \rightarrow \infty} \zeta_{q}^{1 / q}=+\infty
$$

и

$$
\sum_{j=1}^{\infty} \frac{1}{\inf _{q \geqslant j}\left(M_{q} / \zeta_{q}\right)^{1 / q}}<\infty .
$$

Поскольку $R+\varepsilon_{1} / 2<r_{1}+r_{2}$, из [5; $\left.\S 9\right]$ получаем, что существует вещественнозначная функция $f_{1} \in V_{r}\left(B_{R+\varepsilon_{1} / 2}\right), r=r_{1}, r_{2}$, такая, что

$$
\int_{B_{R+\varepsilon_{1} / 2}}\left|f_{1}(x)\right| d x=1 .
$$

Из (26) и [5; следствие 1 из теоремы 2, лемма 6] вытекает, что $\operatorname{supp} f_{1} \cap B_{r_{1}} \neq \varnothing$. Тогда в некотором открытом шаре $B$ с центром $x_{0} \in B_{r_{1}}$ и радиусом $\delta<R-r_{1}$ функция $f_{1}$ не обращается в нуль, т.е. сохраняет знак. Пусть $\varepsilon_{2}=\min \left(\delta, \varepsilon_{1} / 2\right)$ и $\varepsilon=\left(\varepsilon_{1}-\varepsilon_{2}\right) / 2$. Рассмотрим ненулевую неотрицательную функцию $u_{1} \in C^{\infty}\left(\mathbb{R}^{1}\right)$ с носителем в $\left[0, \varepsilon_{2} /(2 \sqrt{n})\right]$, удовлетворяющую условию

$$
\left|u_{1}^{(j)}(t)\right| \leqslant \frac{K_{1}^{j+1} M_{j}}{\zeta_{j}}, \quad t \in \mathbb{R}^{1}, \quad j \in \mathbb{Z}_{+},
$$

где постоянная $K_{1}>0$ не зависит от $j$ и $t$. Существование такой функции следует из $(25)$ и [20; теоремы $1.3 .5,1.3 .8]$. Полагая

$$
K=\max _{j \geqslant 1} \frac{K_{1}^{1+j}}{\zeta_{j}}, \quad u(t)=\frac{u_{1}(t)}{K},
$$


имеем $\left|u^{(j)}(t)\right| \leqslant M_{j}$ на $\mathbb{R}^{1}$. Для $x \in \mathbb{R}^{n}$ положим

$$
\psi(x)=\prod_{j=1}^{n} u\left(x_{j}\right)
$$

тогда $\psi \geqslant 0$ и

$$
\operatorname{supp} \psi \subset B_{\varepsilon_{2} / 2} \text {. }
$$

Кроме того, для всех $x \in \mathbb{R}^{n}, \alpha \in \mathbb{Z}_{+}^{n}$ получаем

$$
\left|\left(\partial^{\alpha} \psi\right)(x)\right|=\prod_{j=1}^{n}\left|u^{\left(\alpha_{j}\right)}\left(x_{j}\right)\right| \leqslant \prod_{j=1}^{n} M_{\alpha_{j}} \leqslant M_{|\alpha|} .
$$

Далее, свертка $f=f_{1} * \psi$ определена в $B_{R+\varepsilon}$ и принадлежит $V_{r}\left(B_{R+\varepsilon}\right), r=r_{1}, r_{2}$. Из (28) и (26) следует, что $\left|\left(\partial^{\alpha} f\right)(x)\right| \leqslant M_{|\alpha|}$ для всех $x \in B_{R+\varepsilon}, \alpha \in \mathbb{Z}_{+}^{n}$. Поскольку $\varepsilon_{2} / 2<\delta$, из $(27)$ и определения $f$ имеем $f\left(x_{0}\right) \neq 0$. Таким образом, $f$ удовлетворяет всем требуемым условиям и утверждение 2 ) доказано.

В заключение отметим, что аналогично можно получить подобные теоремы о двух радиусах и для других уравнений типа (1), рассмотренных в [15]-[17].

\section{СПИСОК ЦИТИРОВАННОЙ ЛИТЕРАТУРЫ}

[1] L. Zalcman, "Analyticity and the Pompeiu problem", Arch. Rational Mech. Anal., 47 (1972), 237-254.

[2] I. D. Smith, "Harmonic analysis of scalar and vector fields in $\mathbb{R}^{n "}$, Proc. Cambridge Philos. Soc., 72 (1972), 403-416.

[3] C. A. Berenstein, R. Gay, "A local version of the two-circles theorem", Israel J. Math., 55 (1986), 267-288.

[4] C. A. Berenstein, R. Gay, A. Yger, "Invertion of the local Pompeiu transform", J. Analyse Math., 54 (1990), 259-287.

[5] В.В. Волчков, "Окончательный вариант локальной теоремы о двух радиусах", Матем. сб., 186:6 (1995), 15-34.

[6] C. A. Berenstein, L. Zalcman, "Pompeiu's problem on symmetric spaces", Comm. Math. Helv., 55:4 (1980), 593-621.

[7] M. El Harchaoui, "Inversion de la transformation de Pompeiu locale dans les espaces hyperboliques réel et complexe: cas de deux boules", J. Anal. Math., 67 (1995), 1-37.

[8] J. M. Cohen, M. A. Picardello, "The 2-circle and 2-disk problems on trees", Israel J. Math., 64 (1988), 73-86.

[9] В.В. Волчков, "Решение проблемы носителя для некоторых классов функций", Матем. сб., 188:9 (1997), 13-30.

[10] В.В. Волчков, "Новые теоремы о среднем для решений уравнения Гельмгольца", Матем. сб., 184:7 (1993), 71-78.

[11] В. В. Волчков, "Новые теоремы о двух радиусах в теории гармонических функций", Изв. РАН. Сер. матем., 58:1 (1994), 182-194.

[12] В.В. Волчков, "О множествах инъективности преобразования Радона на сферах", Изв. РАН. Сер. матем., 63:3 (1999), 63-76.

[13] В. В. Волчков, "Об одной проблеме Зальцмана и ее обобщениях", матем. заметки, 53:2 (1993), 30-36.

[14] В.В. Волчков, "Теоремы о двух радиусах на пространствах постоянной кривизны", Докл. РАН, 347:3 (1996), 300-302. 
[15] В. В. Волчков, "Окончательный вариант теоремы о среднем в теории гармонических функций", Матем. заметки, 59:3 (1996), 351-358.

[16] В. В. Волчков, "Новые теоремы о среднем для полианалитических функций", Матем. заметки, 56:3 (1994), 20-28.

[17] Вит. В. Волчков, “Теоремы о среднем для одного класса полиномов”, Сиб. матем. ж., 35:4 (1994), 737-745.

[18] В. В. Волчков, "Теоремы о шаровых средних на комплексных гиперболических пространствах", Доповіді НАН Украіни, 2000, № 4, 7-10.

[19] Г. В. Бадалян, Квазистепенной ряд и квазианалитические классы функций, Наука, M., 1990.

[20] Л. Хёрмандер, Анализ линейных дифференциальных операторов с частными производными. Т. 1. Теория распределений и анализ Фуръе, Мир, М., 1986.

[21] И. Стейн, Г. Вейс, Введение в гармонический анализ на евклидовых пространствах, Мир, М., 1974.

[22] Н.Я. Виленкин, Специальнъе функиии и теория представлений групп, Наука, М., 1991.

[23] Б. Г. Коренев, Введение в теорию бесселевых функиий, Наука, М., 1971.

[24] С. Хелгасон, Группы и геометрический анализ, Интегральная геометрия, инвариантные дифференциальные операторы и сферические функции, Мир, М., 1987.

\section{В. В. Волчков}

Донецкий национальный университет

E-mail: volchkov@univ.donetsk.ua
Поступило

27.07.2000

Исправленный вариант

21.06.2004 